\title{
PSMA/CD3 Tri-specific T-cell Activating Construct HPN424
}

National Cancer Institute

\section{Source}

National Cancer Institute. PSMA/CD3 Tri-specific T-cell Activating Construct HPN424. NCI Thesaurus. Code C156681.

A recombinant antibody derivative composed of tri-specific T-cell activating construct (T riT AC) directed against the human tumor-associated antigen (TAA) prostate-specific membrane antigen (PSMA; FOLH1) and the CD3 antigen found on T-lymphocytes and an albumin-binding domain, with potential immunostimulating and antineoplastic activities. Upon administration, PSMA/CD3 tri-specific T-cell antibody construct HPN424 targets and binds PSMA on tumor cells and CD3 on cytotoxic T-lymphocytes (CT Ls), thereby bringing PSMA-expressing tumor cells and CT Ls together, which results in the CTLmediated cell death of PSMA-expressing tumor cells. The albumin-binding domain targets and binds to serum albumin, thereby extending the serum half-life of HPN424. PSMA is overexpressed on the surface of metastatic and hormone-refractory prostate tumor cells. 